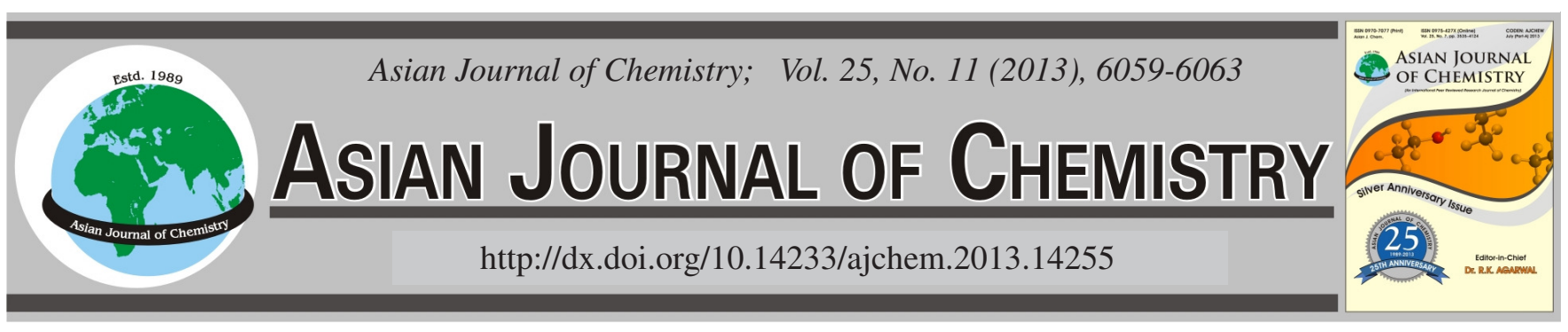

\title{
Comparison of Simultaneous Distillation and Extraction, Static Headspace and Headspace-Solid Phase Microextraction Coupled with GC/MS to Measure the Flavour Components of Tricholoma matsutake
}

\section{Xuan Qin $\mathrm{Chen}^{1}$, Qian Qian Wang ${ }^{1}$, Jun Ming $\mathrm{Li}^{2}$, Ya Qin Wang ${ }^{2}$, Xue Zhong Zhang ${ }^{2}$ and Kun Zhi $\mathrm{Li}^{1, *}$}

${ }^{1}$ Biotechnology Research Center, Faculty of Life Science and Technology, Kunming University of Sciences and Technology, Kunming 650500, P.R. China

${ }^{2}$ Institute of Product Quality Supervision and Inspection, Kunming 650223, P.R. China

*Corresponding author: E-mail: likunzhikm@yahoo.com.cn

\begin{abstract}
Simultaneous distillation and extraction, static headspace assay and headspace-solid phase microextraction combined with GC/MS, were optimized to analyze flavour compounds in Tricholoma matsutake. The optimal conditions for simultaneous distillation and extraction were as following: sample/solution $6: 8$ (w:v), incubation $1 \mathrm{~h}, 90{ }^{\circ} \mathrm{C}$ distillation $2 \mathrm{~h}, 1 \mathrm{~min}$ extraction. The optimal conditions for static headspace assay were $130^{\circ} \mathrm{C}$ equilibrium $30 \mathrm{~min}$. For headspace-solid phase microextraction, the best conditions were $80^{\circ} \mathrm{C}$ equilibrium 5 min, PDMS fibers extraction. 52, 27 and 27 flavour compounds can be identified by the improved simultaneous distillation and extraction, static headspace assay and headspace-solid phase microextraction, respectively. The analytical results from headspace-solid phase microextraction were similar to those from simultaneous distillation and extraction, of which 14 flavour compounds were also found in simultaneous distillation and extraction. Headspace-solid phase microextraction and simultaneous distillation and extraction were partly overlapped. While only 7 compounds from static headspace assay can also be detected in headspace-solid phase microextraction and simultaneous distillation and extraction. Twenty compounds unidentified in headspace-solid phase microextraction and simultaneous distillation and extraction can be detected in static headspace assay. Therefore, we suggested that the complementation and combination of improved static headspace assay and headspace-solid phase microextraction were applied to the analysis of flavour compounds in T. matsutake due to its efficiency and simplicity.
\end{abstract}

Key Words: Static headspace assay, Headspace-solid phase microextraction, Tricholoma matsutake, GC/MS.

\section{INTRODUCTION}

T. matsutake, called songrong jun in China, was one kind of esculent and delicious mushroom species worldwide. T. matsutake benefited human health well due to its cholesterol lowering, antioxidant, immunomodulating, antitumor effects ${ }^{1-3}$. The quality of mushrooms depends on factors such as their aroma, taste, texture and colour, of which the aroma is most critical $^{4}$. GC/MS is the most versatile method for the analysis of volatile aromatic components in the present. The preprocessing methods for GC/MS are decisive factors to the analytical results. Many jobs have been done concerning on the analysis of flavour components of T. matsutake by GC/MS method $^{5-7}$. The results from the predecessors showed that the aromatic components of $T$. matsutake primarily composed a series of $\mathrm{C}_{8}$ components i.e., 3-octanol, 1-octen-3-ol, 1-octanol, (E)-2-octen-1-ol, 3-octanone, 1-octen-3-one, (E)-2-octenal and octanoic acid ${ }^{6,7}$. However, when consulting the references, we found that almost all the researchers utilized simultaneous distillation and extraction as the pre-processing method to analyze the flavour compositions in T. matsutake. Simultaneous distillation and extraction need relative long time and large quantity of sample ${ }^{4-7}$. Static headspace is a simple and fast technique to implement because no sample preparation or solvent is needed. Static headspace is used in food flavour analysis for the extraction of volatile compounds of olive oils, rice and cheeses ${ }^{8-10}$. Headspace-solid phase microextraction (SPME), developed by Arthur and Pawliszyn in 1990, is also a solvent-free sample preparation technique for the extraction of volatile and semivolatile compounds ${ }^{11,12}$. This method, composed of a fused-silica fiber coated with different stationary phases, has been successfully used in the characterization of fragrance of several mushrooms ${ }^{13-15}$. The objective of this paper is to optimize the simultaneous distillation and extraction, static headspace and HS-SPME to compare their applicability to the analysis of volatile compounds of T. matsutake. 


\section{EXPERIMENTAL}

All the fresh samples were bought just before analysis from Chinese Traditional Medical Market, Juhua village, Kunming, Yunnan province, P.R. China.

Simultaneous distillation and extraction (SDE): The samples were cut into pieces $(0.3 \times 0.3 \mathrm{~cm})$. To select the optimal ratio of sample/solution and incubation time, 15, 20, 25, 30 and $40 \mathrm{~g}$ were mixed with $500 \mathrm{~mL}$ water in sealed flask and incubated 1, 2, 4 and 8 h. $40 \mathrm{~mL}$ anhydrous ether was added to another flask to collect the volatile substances. The mixture was distilled at $65^{\circ} \mathrm{C}$ for $0,0.5,1,24,8 \mathrm{~h}$ to select an optimal distillation and extraction time. Then, the collected volatile potions were dried with nitrogen and dissolved with $500 \mu \mathrm{L}$ hexane for GC/MS analysis.

Simultaneous distillation and extraction (SDE): $0.1 \mathrm{~g}$ mushroom was homogenized and immediately sealed in $25 \mathrm{~mL}$ headspace vials with silicone rubber Teflon caps. $5 \mathrm{~mL} \mathrm{0.1,0.2}$ and $0.3 \mathrm{~g} \mathrm{~mL}^{-1} \mathrm{NaCl}$ was added to the vials to examine the effect of $\mathrm{NaCl}$ on the analytical results. To select an optimal equilibrium temperature, vials were equilibrated at 60, 80, 100, 120,140 and $150{ }^{\circ} \mathrm{C}$. To select an optimal equilibrium time, vials were equilibrated for 10, 20, 30 and $60 \mathrm{~min}$.

HS-SPME extraction: $0.1 \mathrm{~g}$ mushroom was homogenized and immediately sealed in $25 \mathrm{~mL}$ headspace vials with silicone rubber Teflon caps. The vial was kept at $60,70,80$ and $90{ }^{\circ} \mathrm{C}$ with continuous internal stirring for $30 \mathrm{~min}$ to determine the optimal equilibrium time; then, the PDSM SPME fiber was exposed to the headspace for 1, 2, 5 and 10 min to select an optimal absorption time. After sampling, the SPME fiber was introduced into the GC injector and was left for $3 \mathrm{~min}$ to allow the analytes thermal desorption.

GC/MS analysis: GC/MS was performed with GCMSQP2010 chromatogram system (Shimadzu) equipped with AOC-5000 automatic static headspace injector (Shimadzu) and DB-5MS(30 m $\times 0.25 \mathrm{~mm}, 0.25 \mu \mathrm{m}$, Agilent) column. Helium $(10 \mathrm{~mL} / \mathrm{min}$ linear speed) was the carrier gas. The injector temperature was set at $180^{\circ} \mathrm{C}$. The oven temperature was maintained at $50^{\circ} \mathrm{C}$ for $5 \mathrm{~min}$ and programmed to $250^{\circ} \mathrm{C}$ at $3{ }^{\circ} \mathrm{C} / \mathrm{min}$. Mass spectrometry analysis was carried out using a Hewlett-Packard mass selective detector model 5973 coupled to the gas chromatograph. The mass spectrometer was operated in the electron impact ionization mode $(70 \mathrm{eV})$, with a scan range of 40 to 400 amu. The ion source temperature was set at $200^{\circ} \mathrm{C}$.

Volatile compounds identification: Volatile compounds were identified by comparing their spectra to those of the Wiley library and also by comparison of their GC Kovats index and retention time to those of standard compounds and data from literature ${ }^{16}$.

Quantitative measurements: The total content of the volatile of each analysis was defined by integrating the peak areas of compounds identified. The relative percentages of individual compounds were calculated from the total contents of volatile on the chromatograms.

\section{RESULTS AND DISCUSSION}

The anhydrous ether was used as the solution to extract the flavour compounds from the $T$. matsutake. To assay the effect of ratio of sample quality/anhydrous ether, the fresh $T$. matsutake samples $(15,20,25,30$ and $40 \mathrm{~g})$ and $40 \mathrm{~mL}$ anhydrous ether were used to allow the sample quality/anhydrous ether was $3: 8,4: 8,5: 8,6: 8,1: 1(\mathrm{~W}: \mathrm{V})$, respectively. As shown in Fig. 1, the total peak area in $40 \mathrm{~g}(1: 1)$ was the largest; however, detail comparison indicated that the nonaromatic compounds $(45 / 192$, data not revealed) in $40 \mathrm{~g}$ was more than that $(47 / 110$, data not revealed) in $30 \mathrm{~g}$, suggesting that the best ration of sample quality/anhydrous ether was 6:8 in simultaneous distillation and extraction. Incubation time is another factor to influence the outcome. Too less time may cause the volatile compounds not to be completely released from the materials. On the other hand, the volatile compounds may vapourize due to long time incubation, resulting in the inaccurate outcome. Appropriate incubation time was necessary for good analytical results. The total peak area of volatile compounds came to a head when incubated in one hour, which indicated one hour incubation was the best for the analysis in simultaneous distillation and extraction (Fig. 2). Extraction time can also influence the analytical outcome like incubation time. As revealed in Fig. 3, the total peak area of volatile compounds amounted to a maximum in $2 \mathrm{~min}$, suggesting $2 \mathrm{~min}$ extraction was the best. Thus, the optimal conditions for simultaneous distillation and extraction were as following: sample/solution 6:8 (w:v), incubation $1 \mathrm{~h}$, $90{ }^{\circ} \mathrm{C}$ distillation $2 \mathrm{~h}, 2$ min extraction.

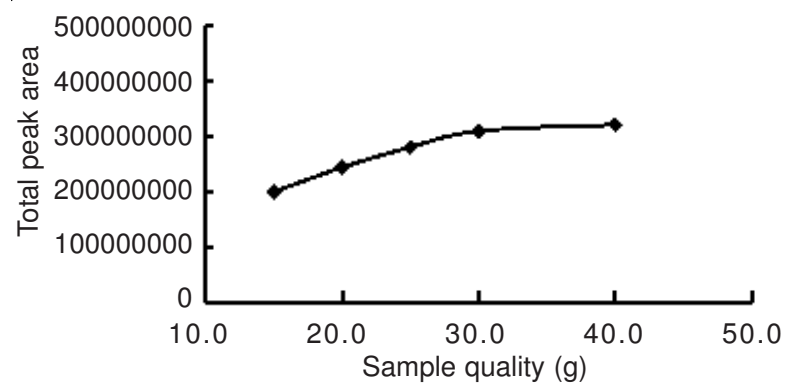

Fig. 1. Effect of sample quality on the total volatile compounds in SDE

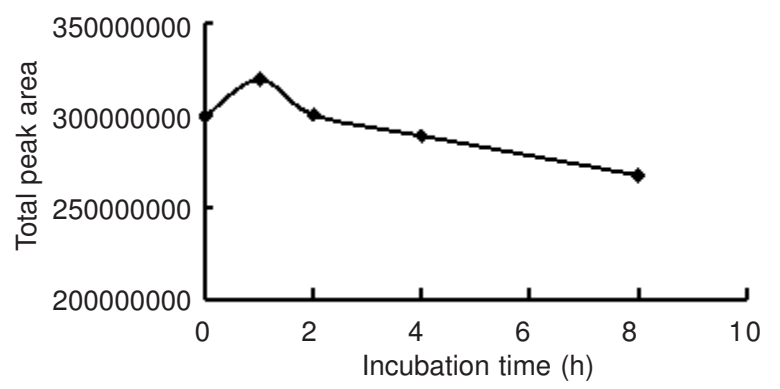

Fig. 2. Effect of incubation time on the total volatile compounds in SDS

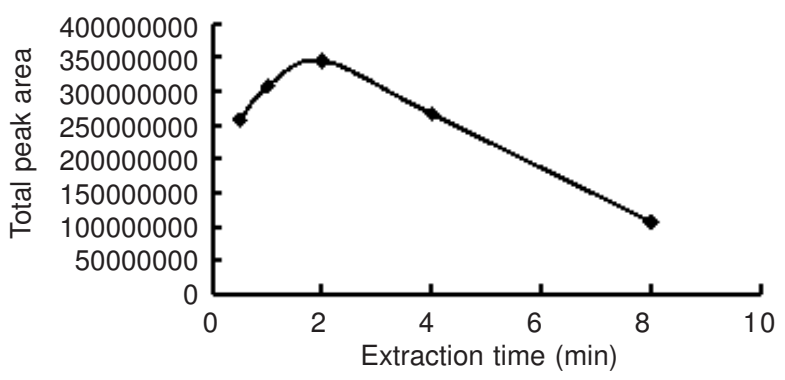

Fig. 3. Effect of extraction time on the total volatile compounds in SDS 
For the static headspace, we selected equilibrium temperature, time and $\mathrm{NaCl}$ concentration as the optimized parameters. Fig. 4 showed that the total peak area of volatile compounds gradually increased with the increase of equilibrium temperature. When the equilibrium temperature arrived at $130{ }^{\circ} \mathrm{C}$ and $140{ }^{\circ} \mathrm{C}$, the total peak area was similar. The detail analysis of spectra indicated that nonaromatic compounds in $140{ }^{\circ} \mathrm{C}$ was more when compared that in $130{ }^{\circ} \mathrm{C}$ (data not revealed). In addition, the higher equilibrium temperature was, the more artificial compounds were produced. It was indicated that the best equilibrium temperature was $130{ }^{\circ} \mathrm{C}$ in static headspace. The volatility of organic compounds is essential for the GC/MS analysis. The total peak area of volatile compounds significantly reduced with the increase of $\mathrm{NaCl}$ concentration (Figs. 5 and 6). One explanation was that $\mathrm{NaCl}$ solution can help to enhance the volatility of polar compounds, while, volatile compounds in T. matsutake chiefly was nonpolar molecular ${ }^{17}$. For the reason, the $\mathrm{NaCl}$ solution was not suggested to be added in static headspace. As revealed in Fig. 7, the total peak area of volatile compounds was increased as the equilibrium time increase. After $30 \mathrm{~min}$, the total peak area almost kept unchangeable, indicating the majority of volatile compounds has vapourized from the materials. The optimal equilibrium time was $30 \mathrm{~min}$. Hence, the optimal conditions for static headspace were $130^{\circ} \mathrm{C}$ equilibrium $30 \mathrm{~min}$, without $\mathrm{NaCl}$.

The total peak area of volatile compounds gradually increased with the increase of equilibrium temperature in the HS-SPME (Fig. 8). The volatility of compounds reached the

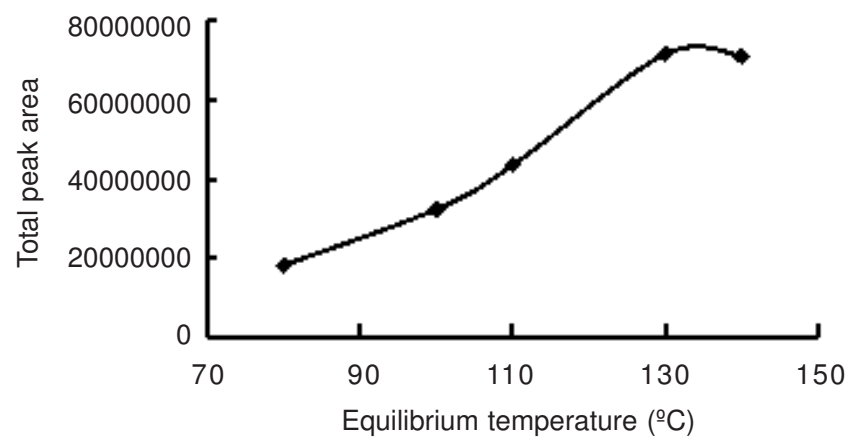

Fig. 4. Effect of equilibrium temperature on the volatile compounds in SHS

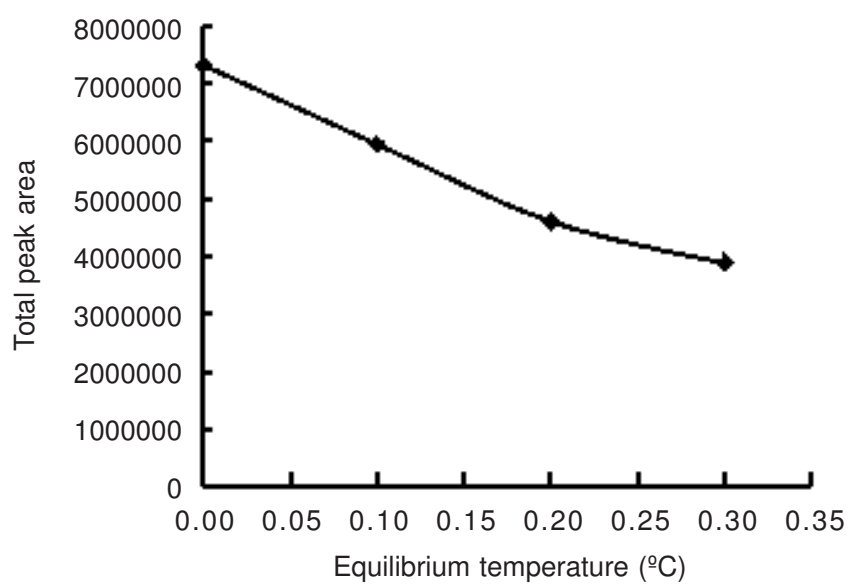

Fig. 5. Effect of $\mathrm{NaCl}$ on the volatile compounds in SHS saturation in $80{ }^{\circ} \mathrm{C}$. Therefore, we selected the $80{ }^{\circ} \mathrm{C}$ as the optimal equilibrium temperature. Fig. 9 showed that the total volatile compounds gradually increased with the increase of equilibrium time. The optimal equilibrium time was $5 \mathrm{~min}$ due to the largest peak area in 5 min.

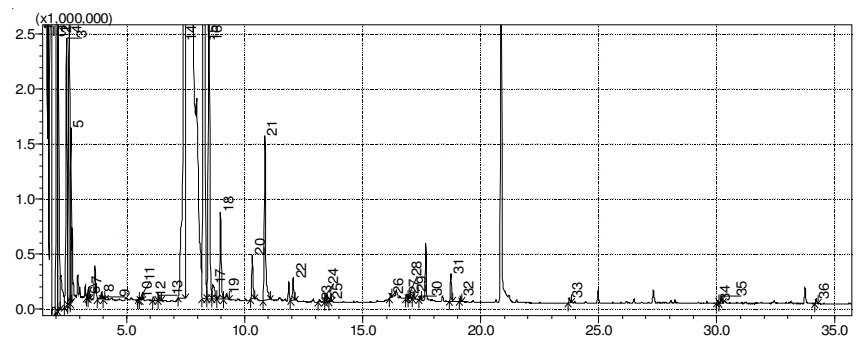

Fig. 6. Comparison of GC spectrum of volatile compounds in SHS with/ without $\mathrm{NaCl}$ extraction red means $0.1 \mathrm{~g} / \mathrm{mL} \mathrm{NaCl}$ in sampling, Black means no $\mathrm{NaCl}$ in sampling

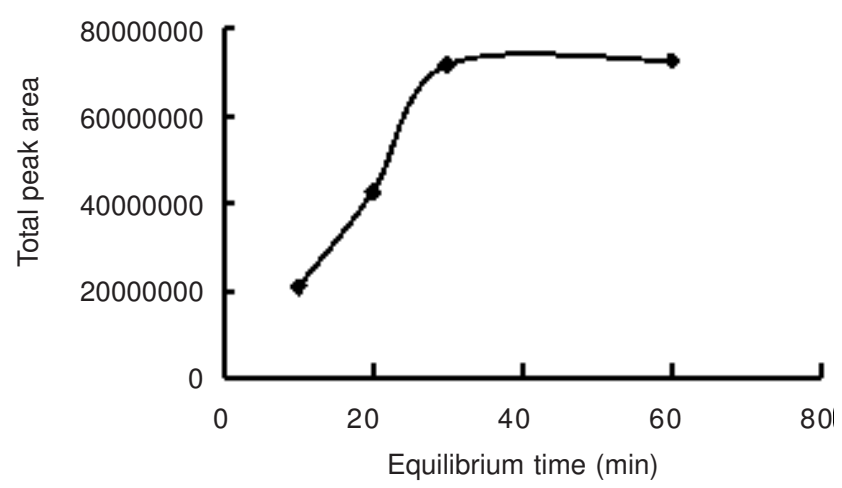

Fig. 7. Effect of equilibrium time on the volatile compounds in SHS

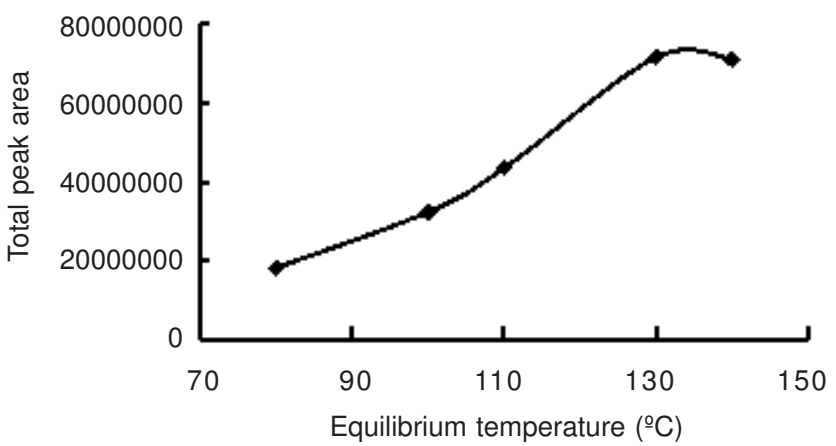

Fig. 8. Effect of equilibrium temperature on the volatile compounds in HS-SPME

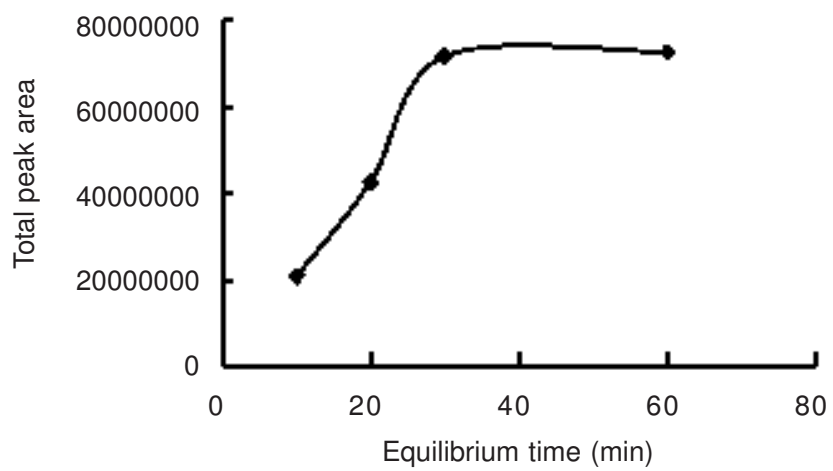

Fig. 9. Effect of equilibrium time on the volatile compounds in HS-SPME 
The GC spectra of T. matsutake essential oil by SDE, SHS and HS-SPME are shown in Figs. 10-12, respectively.

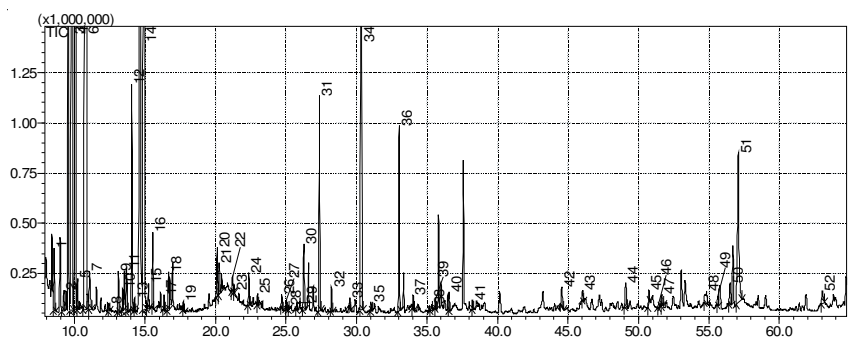

Fig. 10. GC spectrum of T. matsutake essential oil by SDE

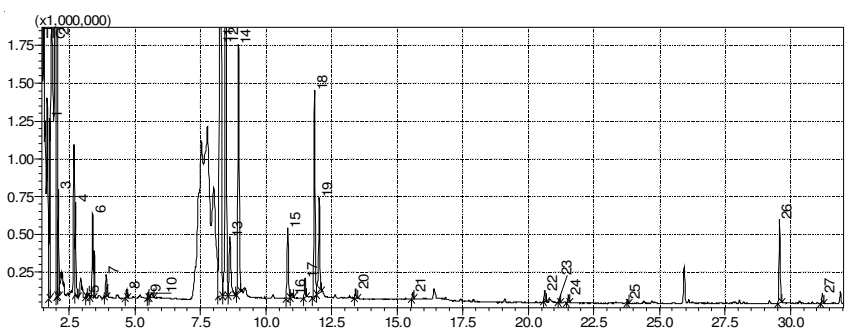

Fig. 11. GC spectrum of T. matsutake essential oil by SHS

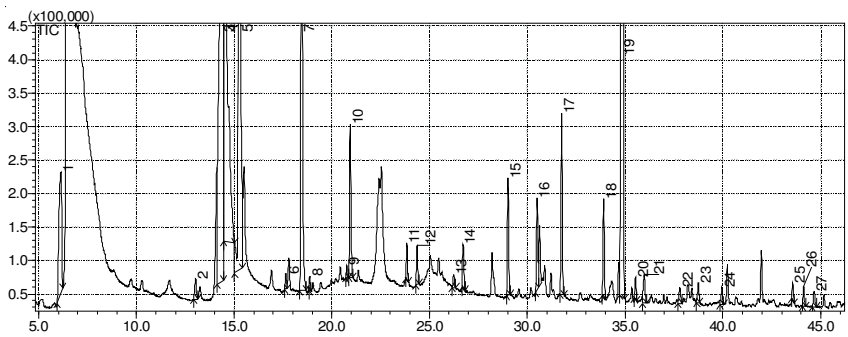

Fig. 12. GC spectrum of T. matsutake essential oil by HS-SPME

52, 27 and 27 flavour compounds can be identified by the improved simultaneous distillation and extraction, static headspace and HS-SPME methods, respectively (Tables 1-3). The main compounds from the three methods are both 1-octylene-3-ol, 3-octanone, 1-octanol and 3-octanol, which were the main aromatic constituents of $T$. matsutake. ${ }^{4-6}$ The volatile aromatic compounds from simultaneous distillation and extraction were more compared with those from static headspace and HS-SPME. But the relative percentage of 1-octylene-3-ol, 3-octanone, 1-octanol and 3-octanol in the three methods are almost the same. More identification of volatile aromatic compounds in simultaneous distillation and extraction was possibly caused by the sample quality differentia used in the three methods rather than the methods differentia. In the simultaneous distillation and extraction, $20 \mathrm{~g}$ sample was used, while $0.1 \mathrm{~g}$ sample was used in static headspace and HS-SPME, which led to more trace compounds accumulation in simultaneous distillation and extraction. It is predicated that appropriate increase sample in HS-SPME and static headspace will brought about more identification of aromatic compounds. In HS-SPME, 14 of 27 compounds can also be found in simultaneous distillation and extraction. This may be due to the specific extraction of PDMS fibers in HS-SPME. HS-SPME and simultaneous distillation and extraction were partly overlapped. In comparison, only 7 compounds, including 1-octylene-3-ol, 3-octanone, 1-octanol, 3-octanol, (E)-2-nonenal, (E,E)-2,4-decadienal and methyl cinnamate in static headspace, can be also found in HS-SPME and simultaneous distillation and extraction. This indicated that the static headspace seemed different from that of simultaneous distillation and extraction and HS-SPME. Some volatile compounds unidentified by HS-SPME and simultaneous distillation and extraction can be detected by static headspace.

\begin{tabular}{|c|c|c|}
\hline \multicolumn{3}{|c|}{$\begin{array}{c}\text { TABLE-1 } \\
\text { LIST OF FLAVOUR COMPOUNDS IN T. matsutake } \\
\text { ESSENTIAL OIL OBTAINED BY SDE }\end{array}$} \\
\hline Peak & Chemical name & Relative content $(\%)$ \\
\hline 1 & Benzaldehyde & 0.41 \\
\hline 2 & 1-Heptanol & 0.14 \\
\hline 3 & 1-Octen-3-ol & 31.26 \\
\hline 4 & 3-Octanone & 14.95 \\
\hline 5 & 2-Pentylfuran & 0.18 \\
\hline 6 & 3-Octanol & 16.43 \\
\hline 7 & Octanal & 0.32 \\
\hline 8 & 2-Ethyl-1-hexanol & 0.03 \\
\hline 9 & Phenylacetaldehyde & 0.21 \\
\hline 10 & (E)-2-Octenal & 0.11 \\
\hline 11 & Hexanal & 0.26 \\
\hline 12 & (E)-2-Nonenal & 1.23 \\
\hline 13 & Acetophenone & 0.07 \\
\hline 14 & 1-Octanol & 14.48 \\
\hline 15 & 1,2-Epoxyethylbenzene & 0.17 \\
\hline 16 & cis-Linalool oxide B & 0.39 \\
\hline 17 & Linalol & 0.07 \\
\hline 18 & Nonanal & 0.22 \\
\hline 19 & Methyl octanoate & 0.05 \\
\hline 20 & Methyl trans-2-octenoate & 0.28 \\
\hline 21 & (E)- 2-Heptenal & 0.25 \\
\hline 22 & 2-Decanone & 0.11 \\
\hline 23 & (Z)-3-Octen-1-ol & 0.01 \\
\hline 24 & (E,E)-2,4-Nonadienal & 0.18 \\
\hline 25 & 3-Phenyl-1-propanol & 0.06 \\
\hline 26 & (E)-2-Decenal & 0.07 \\
\hline 27 & (E)-3-Phenyl-2-propenal & 0.05 \\
\hline 28 & Methyl-3-phenylpropionate & 0.02 \\
\hline 29 & Anethol & 0.03 \\
\hline 30 & (E,E)-2,4-Undecadienal & 0.44 \\
\hline 31 & (E,E)-2,4-Decadienal & 1.13 \\
\hline 32 & (Z)-4-decenal & 0.13 \\
\hline 33 & 8-Undecenal & 0.04 \\
\hline 34 & Methyl cinnamate & 11.99 \\
\hline 35 & (E,Z)-2,4-decadienoate & 0.05 \\
\hline 36 & $\alpha$-Santalene & 1.07 \\
\hline 37 & Ethyl cinnamate & 0.07 \\
\hline 38 & 2-Tridecanone & 0.04 \\
\hline 39 & Pentadecane & 0.05 \\
\hline 40 & Methyl laurate & 0.09 \\
\hline 41 & Docosane & 0.05 \\
\hline 42 & Dihydro-5-octyl-2(3H)-furanone & 0.14 \\
\hline 43 & Henicosane & 0.07 \\
\hline 44 & Carveol & 0.23 \\
\hline 45 & Pentacosane & 0.09 \\
\hline 46 & Tetradecanal & 0.03 \\
\hline 47 & Myrtenyl acetate-FEMA 3765 & 0.10 \\
\hline 48 & Tetracosane & 0.16 \\
\hline 49 & FEMA 2475 & 0.06 \\
\hline 50 & $(5 \alpha, 9 \beta, 10 \beta)$-7-Drimen-11-ol & 0.19 \\
\hline 51 & Hexadecanoic acid & 1.59 \\
\hline 52 & Oxacyclohexadecan-2-one & 0.12 \\
\hline
\end{tabular}




\begin{tabular}{clc}
\hline \multicolumn{3}{c}{ TABLE-2 } \\
\multicolumn{3}{c}{ LIST OF AROMA COMPOUNDS IN T. matsutake } \\
\hline \multicolumn{3}{c}{ EXTRACD BY SHS } \\
\hline 1 & Chemical name & Relative content (\%) \\
2 & 2-Pentanone & 3.67 \\
3 & Pentanal & 8.33 \\
4 & 2-Methylbutanal & 1.78 \\
5 & Tiglaldehyde & 1.54 \\
6 & Caproaldehyde & 0.10 \\
7 & 2-Furaldehyde & 2.39 \\
8 & 1-Hexanol & 0.52 \\
9 & Heptaldehyde & 0.21 \\
10 & 3-(Methylthio-)propionaldehyde & 0.12 \\
11 & 1-Octen-3-ol & 0.20 \\
12 & 3-Octanone & 37.93 \\
13 & 2-Pentylfuran & 11.30 \\
14 & 3-Octanol & 2.71 \\
15 & Phenylacetaldehyde & 10.80 \\
16 & (Z)-3-Hexenylphenylacetate. & 2.36 \\
17 & (E)2-Octenal & 0.18 \\
18 & 1-Octanol & 0.62 \\
19 & Octyl formate & 8.25 \\
20 & Pelargonaldehyde & 3.20 \\
21 & (E)-2-Nonenal & 0.26 \\
22 & 2-Undecanone & 0.16 \\
23 & Tridecanal & 0.34 \\
24 & (E,E)-2,4-Decadienal & 0.07 \\
25 & Methyl cinnamate & 0.22 \\
26 & Nerolidol & 0.11 \\
27 & Tetradecyl aldehyde & 2.36 \\
& & 0.29 \\
\hline
\end{tabular}

\begin{tabular}{clc}
\multicolumn{3}{c}{ TABLE-3 } \\
\multicolumn{3}{c}{ LIST OF AROMA COMPOUNDS IN T. matsutake } \\
\hline Peak & Chemical name & Relative content (\%) \\
\hline 1 & Caproaldehyde & 2.64 \\
2 & (E)-2-Heptenal & 0.26 \\
3 & 1-Octen-3-ol & 27.51 \\
4 & 3-Octanone & 15.47 \\
5 & 3-Octanol, & 17.50 \\
6 & Phenylacetaldehyde & 0.17 \\
7 & 1-Octenal & 6.61 \\
8 & Nonane-1,3-diolmonoacetate & 0.11 \\
9 & Linalol & 0.11 \\
10 & Nonyl aldehyde & 1.59 \\
11 & (E) -2-nonenal & 0.33 \\
12 & Neofolione & 0.38 \\
13 & Capraldehyde & 0.15 \\
14 & (E,E)-2,4-Nonadienal & 0.43 \\
15 & (E)-2-Decenal & 1.13 \\
16 & 2-Decanone & 0.88 \\
17 & (E,E)-2,4-Decadienal & 1.79 \\
18 & 8-Undecenal & 0.92 \\
19 & Methyl cinnamate & 20.42 \\
20 & Hexadecane & 0.17 \\
21 & Dodecyl aldehyde & 0.35 \\
22 & (E)-limoneneoxide & 0.19 \\
23 & (E)-Methyl-2-octenoate & 0.20 \\
24 & Heptadecane & 0.18 \\
25 & Diethyl phthalate & 0.15 \\
26 & Octadecane & 0.19 \\
27 & tetradecyl aldehyde & 0.17 \\
\hline & & \\
\hline
\end{tabular}

\section{Conclusion}

The research presented has demonstrated that all the three methods can be applied to analyze the main aromatic compositions in T. matsutake. Simultaneous distillation and extractionGC-MS is traditional method for aromatic compositions. This method demanded long time sample preparation and large sample quality. Since HS-SPME and simultaneous distillation and extraction are mostly overlapped for the analysis of flavour compounds of T. matsutake, simultaneous distillation and extraction can be replaced by HS-SPME on the basis of appropriate increase of analytical sample. On the other hand, some aromatic compounds of T. matsutake not identified by HS-SPME and simultaneous distillation and extraction can be identified by static headspace. Thus, to better understand the flavour compositions in T. matsutake, we suggested the complementation and combination of static headspace and HS-SPME described in the paper were applied to the analysis of aromatic compounds in T. matsutake.

\section{ACKNOWLEDGEMENTS}

This work was supported by Foundation for Training Adult and Young Leaders of Science and Technology of Yunnan Province (2006PY01-10) and Subsidy Programme of Institute of Product Quality Supervision and Inspection (6393-20070026).

\section{REFERENCES}

1. J.L. Mau, H.C. Lin and S.F. Song, Food Res. Int., 35, 519 (2002).

2. H. Hoshi, Y. Yagi, H. Lijima, K. Matsunaga, Y. Ishihara and T. Yasuhara, J. Agric. Food Chem., 53, 8948 (2005).

3. T. Ebina, T. Kubota, N. Ogamo and K. Matsunaga, Biotherapy, 16, 255 (2002).

4. I.H. Cho, H.J. Namgung, H.K. Choi and Y.S. Kim, Food Chem., 106, 71 (2008).

5. I. Cho, S. Minlee, S.Y. Kim, H.K. Choi, K.O. Kim and Y.S. Kim, J. Agric. Food Chem., 55, 2323 (2007).

6. I.E. Cho, S.Y. Kim, H.K. Choi and Y.S. Kim, J. Agric. Food Chem., 54, 6332 (2006).

7. I.E. Cho, H.K. Cho and Y.S. Kim, Food Chem., 54, 4820 (2006).

8. J.F. Cavalli, X. Fernandez, L. Lizzani-Cuvelier and A. Loiseau, J. Agric. Food Chem., 51, 7709 (2003).

9. T. Sriseadka, S. Wongpornchai and P. Kitsawatpaiboo, J. Agric. Food Chem., 54, 8183 (2006).

10. C. Peres, F. Begnaud and J.L. Berdague, Sens. Actuators B, 87, 491 (2002).

11. C.L. Arthur and J. Pawliszyn, Anal. Chem., 62, 2145 (1990).

12. Z. Zhang and J. Pawliszyn, Anal. Chem., 65, 1843 (1993).

13. P. Guedes De Pinho, B. Ribeiro, R.F. Goncalves, P. Baptista, P. Valentao, R.M. Seabra and P.B. Andrade, J. Agric. Food Chem., 56, 1704 (2008).

14. P. Diaaz, F.J. Senoraans, G. Reglero and E. Ibanez, J. Agric. Food Chem., 50, 6468 (2002).

15. N. Stoppacher, B. Kluger, S. Zeilinger, R. Krska and R. Schuhmacher, J. Microbiol. Meth., 81, 187 (2010).

16. M. Rychlik, P. Schieberle and W. Grosch, Compilation of Odor Thresholds, Odor Qualities and Retention Indices of Key Food Odorants; Deutsche Forschungsanstalt fur Lebensmittelchemie: Garching, Germany (1998).

17. J.C. Florez-Menendez, M.L. Fernandez-Sanchez, J.E. Sanchez-Uria, E. Fernandez-Martinez and A. Sanz-Medel, Anal. Chem. Acta, 415, 9 (2000). 